\title{
Gift der 100 therapeutischen Möglichkeiten
}

Mit der Botulinumtoxin-Therapie hat man mittlerweile 25 Jahre Erfahrung. Aus über 100 möglichen Anwendungen wählte Professor Gerhard Reichel, vom Kompetenzzentrum für Bewegungsstörungen, Paracelsusklinik Zwickau, unter anderem das Thema Augenlid für seine Ausführungen während des 4. Deutschen Botulinumtoxin-Kongresses in München.

Was etwa die etablierten Indikationen bei gestörter Lid-Motorik angeht, kommt es dem Experten zufolge darauf an, genau zu differenzieren: So ist Botulinumtoxin A (BTX A) beim palpebralen Blepharospasmus, gekennzeichnet durch eine dystone Aktivität in der Pars palpebralis $\mathrm{m}$. orbicularis oculi im EMG, eine exzellente Option. Oft damit verwechselt wird eine gänzlich andere Störung: Gelingt es einem Patienten nicht, das Lid offen zu halten, obwohl der Lidschlussmuskel entspannt ist, liegt eine Apraxie der Lidöffnung vor. Ursächlich ist hier die Unfähigkeit, den Lidheber zu aktivieren. Manuell kann das Lid bei diesen Patienten einfach geöffnet werden - bis zur nächsten Kontraktion des M. orbicularis oculi. In diesen Fällen ist keine Abhilfe durch BTX zu erwarten.

Weist das Elektromyogramm eine synchrone Aktivität in Augenringmuskel und Lidheber aus, liegt also eine gestörte antagonistische Inhibition vor, kann der Lidkrampf durch die BTX-Behandlung reduziert, aber nicht aufgehoben werden. Um - etwa bei Lagophthalmus - den Lidschluss $\mathrm{zu}$ verbessern, wird mitunter Gold oder Platin in das Oberlid implantiert. Dabei werde übersehen, so der Experte, dass in Rückenlage das schwere Metall das Lid wieder aufzieht. Die BTX-Injektion in den Lidhebermuskel sei eine elegante Variante, eine protektive Ptosis zu erzeugen. Bei einer durchschnittlichen Dauer von drei Monaten sei die so induzierte Ptosis auch eine geeignete Option bei Hornhauterosionen oder -ulzera.

Waldtraud Paukstadt, freie Medizinjournalistin

Satellitensymposium „Herausforderung Therapiezufriedenheit mit Botulinumtoxin" im Rahmen des 4. Deutschen Botulinumtoxin-Kongresses, München, 2.3.2013; Veranstalter: Merz Pharmaceuticals

\section{Individualisierte Therapie mit Ziel Lebensqualität}

Im September letzten Jahres erschien die neue Leitlinie zu Diagnose und Therapie der Parkinsonsyndrome [Eggert $\mathrm{K}$ et al. S2k-Leitlinie Parkinson-Syndrome Diagnostik und Therapie. Stand 9/2012 AWMF-Register Nr.030/010]. Welche Auswirkungen sie im klinischen Alltag hat, diskutierten Experten anlässlich des Parkinson-Kongresses 2013.

Leitlinien sind begründet auf der Evidenz aus klinischen Studien mit Patientengruppen, die meist nicht typisch sind für klinische Populationen. „Von meinen Patienten könnten höchstens $30 \%$ an solchen Studien teilnehmen“, gab Professor Jens Volkmann, Direktor der Neurologischen Klinik der Universität Würzburg, zu. Die Komorbiditäten einer überwiegend geriatrischen Population und eine sich dynamisch über die Zeit verändernde Erkrankung werden in Studien kaum erfasst. Im Alltag ist daher eine individuelle Therapie mit dem Ziel des Erhalts der Lebensqualität notwendig, für die Leitlinien bestenfalls einen Handlungsrahmen anbieten können. Berücksichtigt werden müssen dabei Alter, Komorbidität, Krankheitsdynamik, Leitsymptome, kognitiver Status und Persönlichkeit. „Objektive Marker für eine individualisierte Therapie haben wir nicht, wir sind auf Erfahrungswerte angewiesen“, betonte Volkmann.

\section{Frühe Therapie erhält die Lebensqualität}

Eine frühe Therapie des Parkinson-Syndroms wirkt sich günstig auf die Lebensqualität aus, wie eine Studie zeigte, in der unbehandelte Patienten mit einer frühen Parkinson-Erkrankung einen Verlust an Lebensqualität erlebten, der bei mit Rasagilin (Azilect ${ }^{\circledR}$ ) behandelten Patienten nicht zu beobachten war [Grosset D et al. J Neurol Neurosurg Psychiatry 2007; 78: 465-9]. Die Leitlinien empfehlen den MAO-B-Hemmer als eine Option für die frühe Therapie. „Das kann die Notwendigkeit von L-Dopa herauszögern“, betonte Volkmann. In der Kombination verlängert Rasagilin in einer späteren Krankheitsphase zusätzlich zu L-
Dopa zudem die On-Zeiten tagsüber, wobei der MAO-B-Hemmer auch bei fortgeschrittener Erkrankung vergleichbar verträglich ist wie Placebo [Rascol O et al. Lancet 2005; 365:947-54]. Auch ein positiver Effekt auf das Freezing wurde in dieser Studie festgestellt. Zudem gibt es Hinweise auf günstige Effekte auf nicht-motorische Symptome wie Schmerz, Apathie, Schlafstörungen und Depressionen, wie Professor Alexander Storch, stellvertretender Direktor der Klinik für Neurologie der Universität Dresden, berichtete. Dabei habe Rasagilin auch positive Wirkungen auf Aufmerksamkeit und andere exekutive Funktionen und die Lebensqualität.

Friederike Klein, freie Medizinjournalistin

Experten-Roundtable Parkinson „Kombinationstherapie im Praxischeck: 180 Tage neue Parkinson-Leitlinie", Deutscher Parkinson-Kongress, Würzburg, 14.3.2013. Veranstalter: TEVA Pharma 